\section{PHARMACOGNOSY}

\section{Sorcery 10 Compuler}

from a Correspondent

Plants have been extremely important sources of therapeutic agents and will become increasingly more so in the future. This was the message from the joint meeting of the Phytochemical Society and the Pharmaceutical Society of Great Britain on plant constituents of pharmacological interest held at Chelsea College, London, on January 5.

Some people may have thought of pharmacognosists as witch doctors in that their methods of selection of plants for study have relied to some extent on folklore and ancient custom. In an attempt to meet this criticism, Professor N. R. Farnsworth (University of Illinois) described a computerized survey technique which has been developed to automate the selection of likely species for pharmacological screening. The method involves data retrieval by computer of all aspects of knowledge of natural products including folklore, analyses of constituents and structureactivity and screening tests. The computer is asked to provide a list of plants which have been reported to possess some of the pharmacological properties desired. A complex scoring system is set up to eliminate plants with undesirable characteristics, plants known to contain the wrong sort of constituent and plants which have received a great deal of attention previously. The resulting list of species provides a basis for a reasonably short screening program.

Although the participants at the meeting were perhaps not entirely convinced by the scheme, the evidence put forward for its success was astounding. In a program carried out at Illinois to look for a new central nervous system (CNS) depressant, the computer came up with some 475 plants with possible activity. The arbitrary scoring system reduced this list to a mere twenty species, fourteen of which have been investigated at the present time. Even as a crude 5 per cent solution in water, the active principles of at least two of these plants possess CNS depressant activity which is greater than twenty times the activity of pentobarbitone: these particular species have received only scant scientific attention to date. It seems that at least seventy types of biological activity can be coded for using this method, and an important future is predicted for this type of work: future results will be noted with great interest.

The meeting heard a variety of other contributions which ranged from a discussion of the chemistry, pharmacology and structure-activity correlations of hashish constituents by Professor F. Korte (University of Bonn), to summaries of the chemotaxonomy and pharmacology of the solanaceous

alkaloids by Dr W. C. Evans (University of Nottingham) and of the mitragyna alkaloids by Professor E. J. Shellard (Chelsea College, London).

Perhaps the most exciting area of current research was that reported by Dr K. Jewers (Tropical Products Institute, London) concerning the study of a whole spectrum of biological activity shown by lactone constituents of various tropical plants. Studies in vivo in mice of extracts from several species of Goniothalamus growing in peat swamps of Sarawak have revealed the presence of a compound which displays CNS activity. The active principle, goniothalamin, has been isolated and it has been shown to be structurally related to kawain (an active constituent of the CNS active drug kava, an interesting account of which was given by Professor $\mathbf{R}$. Hansel of the Free University, Berlin). An important property of goniothalamin is its marked antifungal activity (minimum inhibitory concentration 7-30 $\mu \mathrm{g} / \mathrm{ml}$.), and research is at present in hand on the preparation of derivatives of this $\alpha$-pyrone for further pharmacological screening. A second important aspect involved pharmacological studies on limonoid bitter principles. Recent screening of methyl angolensate and products of its thermal degradation indicates interesting analgesic activity worthy of much further research.

Reviewing recent developments in antibiotic research, $\mathrm{Mr}$ B. Lynn (Beecham Research Laboratories) emphasized that major advances in the next few years are likely to proceed from structural modification of existing agents rather than by the introduction of completely novel compounds.

\title{
Blast Waves and the Galaxy
}

THE discovery in 1957 that there is a flow of gas outwards from the centre of the galaxy and the increasingly compelling evidence of violent occurrences in galactic nuclei has stimulated several studies of the kinematics of interstellar gas. The basic phenomena that have to be taken into account are the existence of a central disk-shaped galactic nucleus, of radius about $800 \mathrm{pc}$, which rotates rapidly but does not expand appreciably, the expanding arma relatively dense region of neutral hydrogen about $3 \mathrm{kpc}$ from the galactic centre-which has an outward velocity of $53 \mathrm{~km} \mathrm{~s}^{-1}$ superimposed on the galactic rotation, and the irregular distribution of less dense gas between the nucleus and $3 \mathrm{kpc}$ which has outward velocities of up to $200 \mathrm{~km} \mathrm{~s}^{-1}$.

A further contribution to the discussion is made in next Monday's Nature Physical Science (January 24) by L. P. Rossner of Brown University, who points out that a blast wave originating from an explosion at the

\section{COSMOLOGY}

\section{Another QSO-Gulaxy Pair}

by our Cosmology Correspondent

MORE evidence of a possible association between galaxies and quasars, and another blow to the cosmological interpretation of quasar redshifts, has been provided by the identification of the optical object associated with the radio source of $3 \mathrm{C} 455$ as a QSO. This object lies just 23" away from the galaxy NGC 7413, which was first identified as the optical counterpart of $3 \mathrm{C} 455$. But the redshift of the QSO has been measured as 0.543 , whereas the light from NGC 7413 is shifted by only $z=0.0332$ (Arp, E. M. Burbidge, Mackay, and Strittmatter, Astrophys. J. Lett., 171, L41 ; 1972).

The SO galaxy NGC 7413 was suggested as a likely candidate for the radio source in 1965. Improved radio positions showed, however, that the galaxy lies roughly $20^{\prime \prime}$ south-west of the radio position. It is now clear that the radio position coincides with a blue object (Elsmore and Mackay, Mon. Not. Roy. Astron. Soc., 146, 361; 1969). Arp et al. have studied plates and spectra of this object made with the Hale 200-inch and the Lick 120-inch telescopes. The blue object appears stellar, with a magnitude between 19.5 and 20 , and although there is no visible connexion between this object-now identified as a QSO because of its appearance-and the galaxy, there is photographic evidence that NGC 7413 is asymmetric, and that the luminous matter forming its major axis extends into the quadrant adjacent to the QSO.

By itself this observation could not galactic centre (the basis of one suggested explanation of the outward gas flow-see, for example, J. H. Oort, Trans. Internat. Astron. Union, XIIA, 789 ; 1964) would be modified because of the gravitational acceleration towards the centre and because of the conservation of angular momentum of the material propelled by the wave. $\mathrm{He}$ finds that as a result of this modification some of the material carried along by the blast would fall back towards the galactic centre.

The behaviour of the velocity and pressure profiles with time, as predicted by Rossner, indicates the formation of a "tail shock" behind the shock proper. As the forces acting on the gas are "equivalent to tying down each fluid element to its initial position by a nonlinear spring", Rossner therefore concludes that the conditions necessary for the formation of a central condensation (the nuclear disk at the galactic centre) could very well have existed according to a model such as the one he considers. 